Z. klin. Chem. u. klin. Biochem.

7. Jg., S. 374-378, Juli 1969

\title{
Untersuchungen zum katabolen Mucopolysaccharid-Protein-Stoffwechsel in menschlichen Organen
}

\author{
Von D. Plati ${ }^{1}$ ) und U. Stein \\ Medizinische Kliniken und Polikliniken der Justus-Liebig-Üniversität Gießen (Direktorium: Prof. Dr. H. J. Dengler, \\ Prof. Dr. H. A. Kïhn, Prof. Dr. H. G. Lascb) und Mediqinische Klinile der Universität Freiburg \\ (Direktorium: Prof. Dr. G.W. Löbr, Prof. Dr.W. Gerok)
}

(Eingegangen am 5. Mai 1969)

In der vorliegenden Arbeit wurden in epithelialen und mesenchymalen Geweben vom Menschen die Aktivitäten der Glycosaminoglycano-Hydrolasen: Hyaluronidase, $\beta$-Glucuronidase und $\beta$-Acetylglucosaminidase und die der Peptidhydrolasen: Kathepsin D und saure Carboxypeptidase gemessen. Die höchsten Aktivitäten fanden sich in Thymus, Leber, Milz und Nebenniere. Geringe Aktivitäten wiurden im subkutanen Bindegewebe und im Herzen gemessen. Die synergistische Wirkung der 5 lysosomalen Enzyme am MucopolysaccharidProteinkomplex wurde diskutiert.

\section{Studies on the Catabolic Mucopolysaccharide-Protein Metabolism in Human Organs}

In this investigation the activity of the glycosaminoglycan hydrolases: hyaluronidase, $\beta$-glucuronidase, $\beta$-acetylglucosaminidase and the activity of the peptide hydrolases: cathepsin $D$ and "acid" carboxypeptidase, were measured in human organs. The highest activities were found in the thymus, liver, spleen and suprarenal organ. Low activities were found in the connective tissue of the skin and in the heart: The synergistic mechanism of the five lysosomal enzymes on the mucopolysaccharidprotein complex was discussed.

Der Stoffaustausch zwischen Zelle und Blutgefäßsystem erfolgt stets durch die Interzellularsubstanz, die je nach Lokalisation und Funktion in ihrer Zusammensetzung quantitative und qualitative Unterschiede aufweist. Bei der chemischen Analyse findet man sulfatierte Polyuronide (Chondroitin-4-Sulfat, Chondroitin-6-Sulfat, Dermatansulfat und Heparitinsulfat), Polyuronide (Hyaluronsäure, Chondroitin), Polysulfate (Keratansulfat) und die Skleroproteide Kollagen und Elastin (1). Die Chondroitinsulfate liegen jedoch im Gewebe nicht in freier Form vor, sondern sind konvalent an Proteine gebunden. Nach MATHEw (2) ist im Rindernasenknorpel eine zentrale Proteinkette mit bis zu 60 Chondroitinsulfatketten verbunden. Inzwischen konnten auch aus anderen $\mathrm{Ge}$ weben Chondroitinsulfat-Proteinkomplexe nachgewiesen werden (4-6). Durch die Bindung der sauren Glycosaminoglycane an Proteine gewinnen die Chondroitinsulfate neue Eigenschaften, die im wesentlichen physiko-chemischer Natur sind. So bilden konzentrierte Chondroitinsulfat-Proteinlösungen durch Quer- und Längsverbindungen ein dreidimensionales Gitter, in dessen Innerem Wasser immobilisiert ist. FrImMER und BudDecke (7) konnten an Modellversuchen zeigen, daß diesen Chondroitinsulfat-Proteinkomplexen eine wesentliche Bedeutung für Permeabilitätsvorgänge zukommt. Diese experimentellen Arbeiten weisen daraufhin, daß auch im lebenden Organismus den interzellulär gelegenen Chondroitinsulfat-Proteinkomplexen eine wichtige Aufgabe in derSteuerung des Transportmechanismus zurZelle zukommt. Ein ungestörter Ablauf dieser Vorgänge ist jedoch nur dann möglich, wenn Synthese und Abbauvorgänge in einem Gleichgewicht stehen. Mittels radio-

1) Mit Unterstützung der Deutschen Forschungsgemeinschaft. aktiv markierter Substanzen $\left({ }^{35} \mathrm{SO}_{4},{ }^{14} \mathrm{C}=\right.$ Acetat, ${ }^{14} \mathrm{C}$ Glucose und ${ }^{14} \mathrm{C}$-Serin) wurde die Synthese saurer Mucopolysaccharid-Proteinkomplexe untersucht (8-12).

In der vorliegenden Arbeit sollte nun versucht werden, den Aktivitätsnachweis derjenigen Enzyme zu erbringen, die am Abbau der Mucopolysaccharid-Proteinkomplexe beteiligt sind, um damit einen gewissen Einblick in den katabolen Mucopolysaccharid-Proteinstoffwechsel epithelialer und mesenchymaler menschlicher Gewebe zu gewinnen.

\section{Methodik}

In 13 menschlichen - makroskopisch unauffälligen - Leichenorganen wurde die Aktivität der Hyaluronidase (systematischer Name: Hyaluronat-Glycanohydrolase, EC 3.2.1.35), in 14 Organen wurden die Aktivitäten der $\beta$-Glucuronidase (systematischer Name: $\beta$-D-Glucuronid-Glucuronohydrolase, EC 3.2.1.31) und $\beta$-Acetylglucosaminidase ' (systematischer Name: $\beta$-2-Acetamido2-desoxy-D-glucosid-Acetamidodesoxyglucohydrolase, EC 3.2.1. 30), in 12 Organen wurde die Aktivität der ,sauren“ Carboxypeptidase (EC 3.4.2.) $)^{2}$ ) und in 9 dieser Organe die bei pH 3,2 optimal Hämoglobin-spaltende Kathepsin (EC 3.4.4.23) ${ }^{2}$ ) Aktivität gemessen.

Die Organproben wurden stets direkt nach der Sektion entnommen - pro Organgruppe durchschnittlich 25 Organproben und zunächst bei $-25^{\circ}$ eingefroren. Die eingefrorenen Organe wurden durch einen elektrischen Fleischwolf gedreht, in der dreifachen Menge 0,1M Citrat-Puffer, $\mathrm{pH}$ 4,5 mit 0,15 $\mathrm{M} \mathrm{NaCl}+$ $0,1 \%$ Triton $\mathrm{x}-100$ aufgenommen und unter Kühlung bei $-20^{\circ}$ (Methanolbad) mit einem Ultraturrax ( $F_{2}$. Jahnke und Kunkel, Staufen, Brsg.) homogenisiert. Anschließend wurde das Homogenat bei $-25^{\circ}$ eingefroren. Nach einem weiteren AuftauEinfrierschritt wurde das Homogenat 20 Min. lang in der Kühlzentrifuge $\left(+4^{\circ}\right)$ bei $4000 \mathrm{~g}$ zentrifugiert und der Ubersțand einer

2) In der Enzyme Nomenclature, Elsevier Publ. Comp., 2nd edition, Amşterdam 1965 enthaltenen Liste ist der systematische Name nicht verzeichnet. 


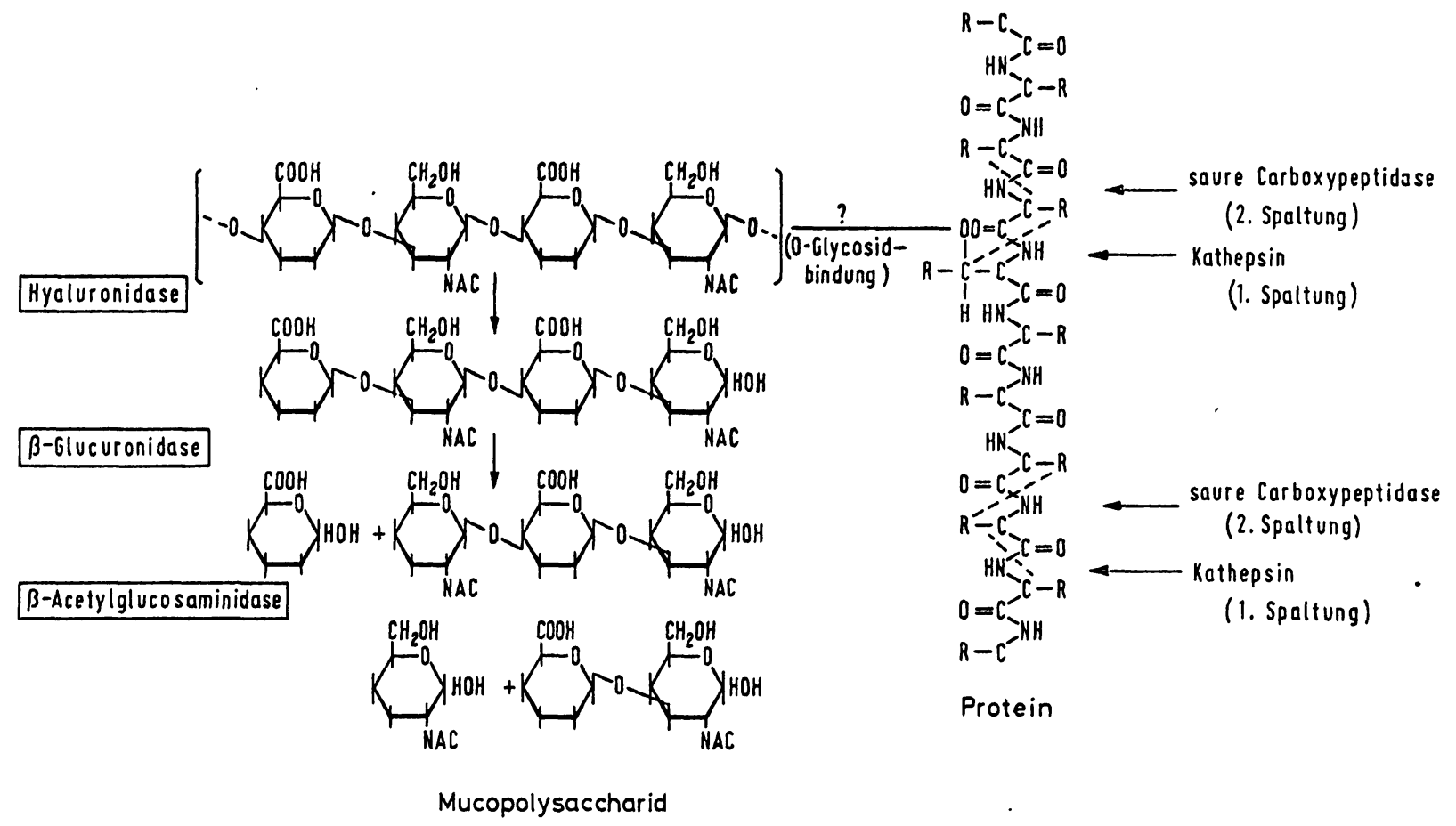

Abb. 1

Scheınatische Darstellung eines Mucopolysaccharid-Protein-Komplexes mit den spezifischen depolymerisierenden Enzymen

fraktionierten Ammoniumsulfat-Fällung unterworfen. Die jewcils durch Zentrifugieren gewonnene Fällung wurde in dest. Wasser aufgenommen und $2 \mathrm{mal} 24 \mathrm{Stdn}$. gegen dest. Wasser dialysiert und anschließend gefriergetrocknet. Allc Operationen wurden bei $+4^{\circ}$ im Kühlraum durchgeführt. Dic Enzymaktivitätsmessungen erfolgten im Homogenat und in den einzelnen Ammoniumsulfatfraktionen. Eine Anreicherung der sauren Carboxypeptidase aus Pankreasgewebe über die Ammoniumsulfatfällung hinaus erfolgte durch $\mathrm{Gcl}$-Filtration an in $0,2 \mathrm{M} \mathrm{NaCl}$-Lösung gequollenem und äquilibriertem Sephadex-G-150. Auf eine Säule von $150 \times$ $1,5 \mathrm{~cm}$ (Volumen: $265 \mathrm{ml}$ ) wurden $45 \mathrm{mg}$ einer 11-30 Gew.\% Ammoniumsulfatfraktion (gelöst in $2-3 \mathrm{~m} / 0,2 \mathrm{M} \mathrm{NaCl}$-Lösung) aufgetragen und mit $0,2 \mathrm{M} \mathrm{NaCl}-\mathrm{L}$ ösung eluiert. Die Protcinbestimmung erfolgte mit der Methode nach WeICHSELbaum (13) bzw. LowrY (14). Die Hyaluronidase-Aktivität wurde durch Messung der freigesetzten $\mathrm{N}$-Acetylglucosamin-Endgruppen bestimmt (15). Als Substrat dienten Hyaluronsäure und Chondroitin-6-Sulfat. Aktivitätsangabe in $E_{546} \mathrm{~nm} / \mathrm{mg}$ Protein/16 Stdn. Inkubationszeit bei $37^{\circ}$. Der Nachweis der $\beta$-Glucuronidase- Aktivität erfolgte an dem Substrat Phenolphthaleinglucuronid nach der Methode von Bernfeld und Fishman (16). $1 \mathrm{mU}=$ $1 \mu \mathrm{Mol}$ freigesetztes Phenolphthalein/mg Protein/Min. bei $37^{\circ}$. Zur Bestimmung der $\beta$-Acetylglucosaminidase-Aktivität wurde das Substrat $\beta$-Phenyl-N-Acetyl-D-glucosaminid verwandt (17). $1 \mathrm{mU}=1 \mu \mathrm{Mol}$ frcigesetztes Phenol pro mg Protein/Min. bei $37^{\circ}$. Die Aktivitätsmessung der sauren Carboxypeptidase erfolgte nach der bei STEIN, WEBER, REICH und BudDECKE $(18,19)$ angegebenen Methodik. Als Substrat wurde L-Seryl-L-Leucyl-LLeucin (Ser-Leu-Leu) verwandt. Die Bestimmung der Carboxy'peptidase- $A$-Aktivität mit den beiden Substraten Benzyloxycarbonyl-Glycyl-Phenylalanin (Z-Gly-Phe, Hersteller: Fa. Mann, New York) und Ser-Leu-Leu erfolgte wie die der sauren Carboxypeptidase; $1 \mathrm{mU}=1 \mu \mathrm{Mol}$ freigesetztes Leucin bzw. Phenylalanin/mg Protein/Min. bei $37^{\circ}$. Die Katbepsin-Aktivität wurde mit denaturiertem Hämoglobin nach der Methode von ANson (1) bestimmt; $1 \mathrm{mU}=1 \mu \mathrm{Mol}$ freigesetztes Tyrosinäquivalent $/ \mathrm{mg}$ Protein/Min. bei $37^{\circ}$. ...

\section{Ergebnisse}

In Vorversuchen konnte durch unterschiedlich lange Lagerung verschiedener Tier-Organe bei Zimmer-,
Kühlraum-, Gefrierschrank- und Brutschranktemperatur ein signifikanter Aktivitätsverlust für die Dauer der Lagerung der Leichen ausgeschlossen werden $(21,22$.$) .$

Tab. 1

Hyaluronidase-Aktivität (Extinktion/mg Protein bei $546 \mathrm{~nm}$ ) in verschiedenen Organen des Menschen $\left(O+O^{\circ}\right)$. Fraktionierte Ammoniumsulfat-Fällung.

Enzymkonzentration/Inkubationsmedium: 1,5 mg. Substratkonzentration: 2,5 mM Hyaluronat-Lösung. Inkubationszeit: $16 \mathrm{St}$. Inkubationstemperatur: $37^{\circ}$.

\begin{tabular}{|c|c|c|c|c|c|}
\hline & $\begin{array}{l}\text { Reinigh } \\
\text { Fraktionierte }\end{array}$ & $\begin{array}{l}\text { ngsschirit } \\
\left.\mathrm{NH}_{4}\right)_{2} \mathrm{SO}\end{array}$ & -Fällung & & \\
\hline & Homogenat & $\begin{array}{c}0-10 \\
\text { Gew. } \%\end{array}$ & $\begin{array}{l}11-30 \\
\text { Gew. } \%\end{array}$ & $\begin{array}{l}31-50 \\
\text { Gew. } \%\end{array}$ & $\begin{array}{l}51-80 \\
\text { Gew. } \%\end{array}$ \\
\hline Gehirn & 0,01 & - & 0,05 & 0,03 & - \\
\hline Herz & 0,005 & - & 0,004 & - & - \\
\hline Hoden & 0,19 & 0,18 & 0,69 & 0,09 & 一 \\
\hline $\begin{array}{l}\text { Hypophyse } \\
\text { Leber }\end{array}$ & $\begin{array}{l}0,09 \\
0,50\end{array}$ & $\overline{025}$ & 0,22 & $\begin{array}{l}0.09 \\
0.04\end{array}$ & $\overline{-}$ \\
\hline Lunge & 0,07 & 0,12 & 0 & 0,03 & 二 \\
\hline ymphknoten & 0,11 & 0,07 & 0,19 & 0,08 & - \\
\hline Milz & 0,66 & 0,50 & 1,18 & 0,09 & - \\
\hline Vebenniere & 0,08 & 0,02 & 0,24 & 0,01 & - \\
\hline Viere & 0,24 & 0,24 & 0,33 & 0,04 & 0,07 \\
\hline Pankreas & & & & & - \\
\hline childdrüse & $\begin{array}{l}0,01 \\
0,02\end{array}$ & 二 & 0,09 & $\overline{-}$ & 一 \\
\hline & & & 0,06 & - & \\
\hline
\end{tabular}

Tab. 2

$\beta$-Glucuronidase-Aktivität ( $\mathrm{mU} / \mathrm{mg}$ Protein) in verschiedenen Organen des Menschen $\left(Q+\infty^{\circ}\right)$. Fraktionierte Ammoniumsulfat-Fällung. Enzymkonzentation/Inkubationsmedium: 500 /g. Substratkonzentration: $0,01 \mathrm{M}$ Phenolphthalein-Glucuronid-Lösung. Inkubationszeit : $\begin{array}{r}4 \text { Stdn. Inkubationstemperatur: } 37^{\circ} \\ \hline\end{array}$

\begin{tabular}{|c|c|c|c|c|c|}
\hline \multicolumn{6}{|c|}{$\begin{array}{c}\text { Reinigungsschritt } \\
\text { Fraktionierte }\left(\mathrm{NH}_{3}\right)_{2} \mathrm{SO}_{4}-\text { Fällung }\end{array}$} \\
\hline & Homogenat & $\begin{array}{l}0-10 \\
\text { Gew. } \%\end{array}$ & $\begin{array}{l}11-30 \\
\text { Gew. } \%\end{array}$ & $\begin{array}{l}31-50 \\
\text { Gew. \% }\end{array}$ & $\begin{array}{l}51-80 \\
\text { Gew. } \%\end{array}$ \\
\hline $\begin{array}{l}\text { Ğehirn } \\
\text { Herz } \\
\text { Hoden } \\
\text { Hypophyse } \\
\text { Leber } \\
\text { Lunge } \\
\text { Lymphknoten } \\
\text { Milz } \\
\text { Nebenniere } \\
\text { Niere. } \\
\text { Pankreas } \\
\text { Schilddrüse } \\
\text { Subcutanes Gewebe }\end{array}$ & $\begin{array}{l}0,18 \\
0,07 \\
1,20 \\
0,18 \\
3,07 \\
0,59 \\
0,19 \\
3,00 \\
0,34 \\
1,38 \\
0,35 \\
0,28 \\
0,02\end{array}$ & $\begin{array}{l}0,05 \\
0,04 \\
0,62 \\
1,07 \\
0,47 \\
0,09 \\
0,64 \\
0,68 \\
1,30 \\
0,17 \\
0,21 \\
\end{array}$ & $\begin{array}{r}0,28 \\
0,19 \\
3,00 \\
0,38 \\
11,02 \\
1,60 \\
0,74 \\
7,40 \\
1,83 \\
1,80 \\
0,98 \\
0,44 \\
0,15\end{array}$ & $\begin{array}{l}0,06 \\
0,05 \\
0,02 \\
0,08 \\
0,30 \\
= \\
= \\
0,10 \\
0,17 \\
0,16 \\
0,21 \\
0,01\end{array}$ & $\begin{array}{l}0,08 \\
0,49 \\
0,02 \\
0,25 \\
= \\
\overline{-} \\
0,10 \\
0,30 \\
0,09 \\
0,12 \\
0,03\end{array}$ \\
\hline
\end{tabular}


Tab. 3

B-Acetylglucosaminidase-Aktivität (mU/mg Protein) in verschiedenen Organen des Menschen $\left(0+\sigma^{\prime}\right)$. Fraktionierte Ammoniumsulfat-

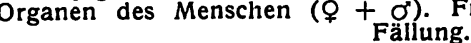

Enzymkonzentration/Inkubationsmedium: $500 \mu \mathrm{g}$. Substratkonzentration 0,025 M $\beta$-Phenyl-N-Acetyl-D-Glucosaminid-Lösung. Inkubationszeit: 1 Std. Inkubationstemperatur: $37^{\circ}$

\begin{tabular}{|c|c|c|c|c|c|}
\hline & \multicolumn{3}{|c|}{$\begin{array}{c}\text { Reinigungsschritt } \\
\text { Fraktionierte }\left(\mathrm{NH}_{4}\right)_{2} \mathrm{SO}_{4}-\text { Fällung }\end{array}$} & \multirow[b]{2}{*}{$\begin{array}{l}31-50 \\
\text { Gew. } \%\end{array}$} & \multirow[b]{2}{*}{$\begin{array}{l}51-80 \\
\text { Gew. } \%\end{array}$} \\
\hline & Homogenat & $\begin{array}{l}0-10 \\
\text { Gew. } \%\end{array}$ & $\begin{array}{l}11-30 \\
\text { Gew. } \%\end{array}$ & & \\
\hline $\begin{array}{l}\text { Gehirn } \\
\text { Herz } \\
\text { Hoden } \\
\text { Hypophyse } \\
\text { Leber } \\
\text { Lunge } \\
\text { Lymphknoten } \\
\text { Milz } \\
\text { Nebenniere } \\
\text { Niere } \\
\text { Pankreas } \\
\text { Schilddrüse } \\
\text { Subcutanes Gewebe }\end{array}$ & $\begin{array}{r}30,18 \\
12,27 \\
91,28 \\
17,84 \\
29,53 \\
19,98 \\
103,70 \\
46,80 \\
93,54 \\
66,47 \\
21,75 \\
7,49 \\
4,87\end{array}$ & $\begin{array}{r}8,57 \\
2,58 \\
58,64 \\
31,20 \\
10,49 \\
60,86 \\
23,99 \\
47,11 \\
39,76 \\
27,73 \\
0,40 \\
\end{array}$ & $\begin{array}{r}49,85 \\
34,08 \\
138,11 \\
64,89 \\
119,72 \\
37,84 \\
141,83 \\
103,38 \\
151,64 \\
118,89 \\
112,42 \\
13,63 \\
18,27\end{array}$ & $\begin{array}{r}9,85 \\
5,42 \\
5,98 \\
25,29 \\
20,52 \\
4,35 \\
16,58 \\
7,65 \\
25,12 \\
38,85 \\
42,86 \\
4,53 \\
4,00\end{array}$ & $\begin{array}{r}9,59 \\
14,69 \\
17,70 \\
\overline{-} \\
14,14 \\
\overline{5,15} \\
17,90 \\
3,86 \\
\overline{9,20}\end{array}$ \\
\hline
\end{tabular}

Mit Ausnahme eines fehlenden Aktivitätsnachweises der Hyaluronidase im Pankreas konnten in allen untersuchten Organen die Aktivitäten der 5 lysosomalen Enzyme nachgewiesen werden. Mit Hilfe einer fraktionierten Ammoniumsulfatfällung (Tab. 1-3) wurden die höchsten Aktivitäten für alle untersuchten Enzyme - mit Ausnahme des Kathepsins - in der 11-30 Gew.\%Fraktion gemessen. Über $60 \%$ der Kathepsin-Aktivität fand sich in der 31-50 Gew.\%-Fraktion. Ein Vergleich der Enzymaktivitäten der untersuchten Organe erfolgte daher in diesen Fraktionen (Abb. 2 u. 3). Die Glycosidasen Hyaluronidase, $\beta$-Glucuronidase und $\beta$-Acetylglucosaminidase zeigten die höchsten Aktivitäten in den Organen des retikulo-histiocytären Systems: Leber, Milz und Thymus. Auffällig sind die hohen Aktivitäten $\operatorname{der} \beta$-Acetylglucosaminidase, der sauren Carboxypeptidase und des Kathepsins in der Nebenniere. Die dritthöchste Carboxypeptidase-Aktivität wurde in der Hypophyse gemessen und lag etwa doppelt so hoch wie in der Leber. Geringe Aktivitäten aller untersuchten Enzyme

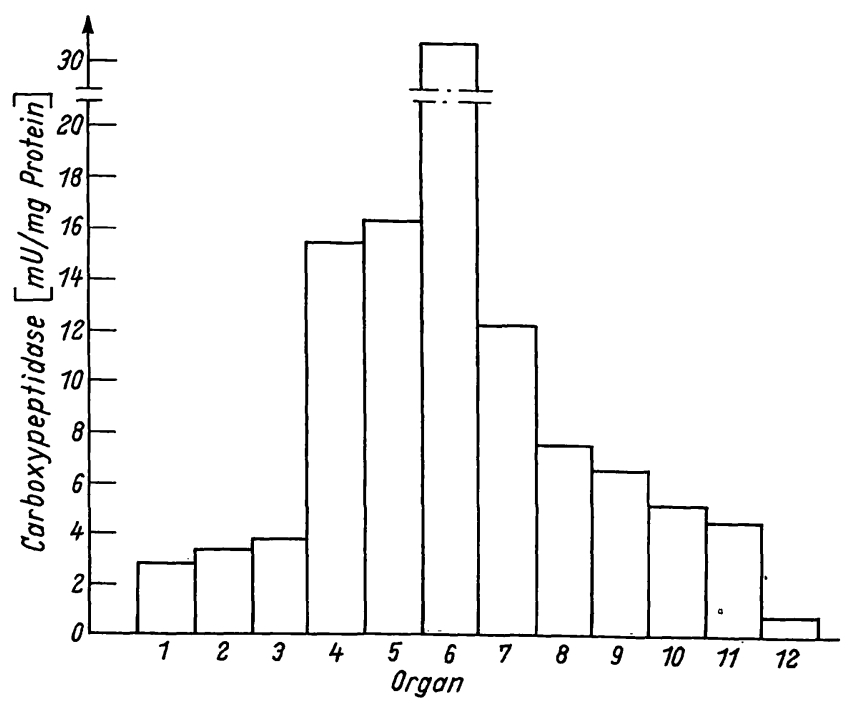

Abb. 2

Vergleich der sauren Carboxypeptidase-Aktivitäten ( $\mathrm{mU} / \mathrm{mg}$ Protein) verschiedener menschlicher Organe $\left(Q+\sigma^{\prime}\right)$ in einer $11-30 \mathrm{Gew} . \%$ $\left(\mathrm{NH}_{4}\right)_{2} \mathrm{SO}_{4}$-Fällung

(1. Lunge, 2. Gehirn, 3. Herz, 4. Hypophyse, 5. Pankreas, 6. Nebenniere, 7. Thymus, 8. Leber, 9. Niere, 10. Milz, 11 . Schilddrüse, 12. subcutanes Bindegewebe)

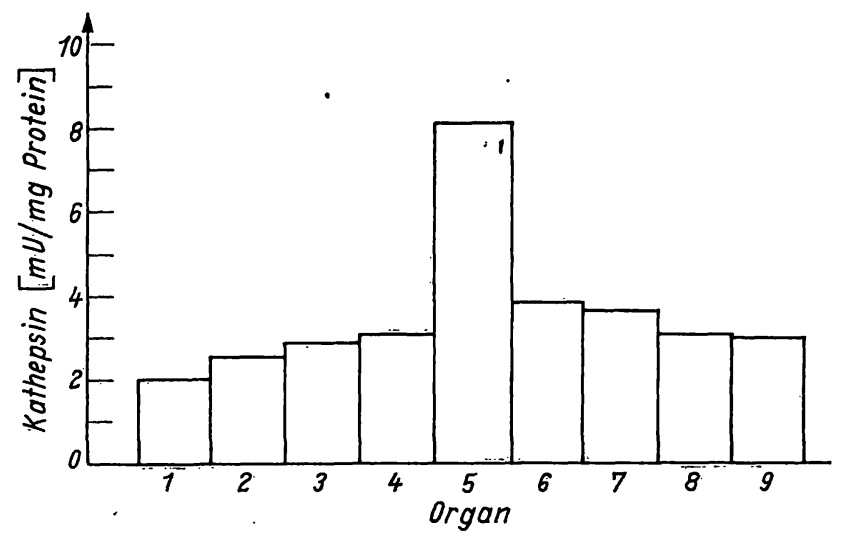

Abb. 3

Vergleich der Kathepsin-Aktivitäten (mU/mg Protein) verschiedener menschlicher Organe $\left(O+\sigma^{\prime}\right)$ in einer $11-30 \mathrm{Gew} \%\left(\mathrm{NH}_{4}\right)_{2} \mathrm{SO}_{4}-$ Fällung (1. Lunge, 2. Gehirn, 3. Herz, 4. Milz, 5. Nebenniere, 6. Pankreas,
7. Leber, 8. Niere, 9. Thymus)

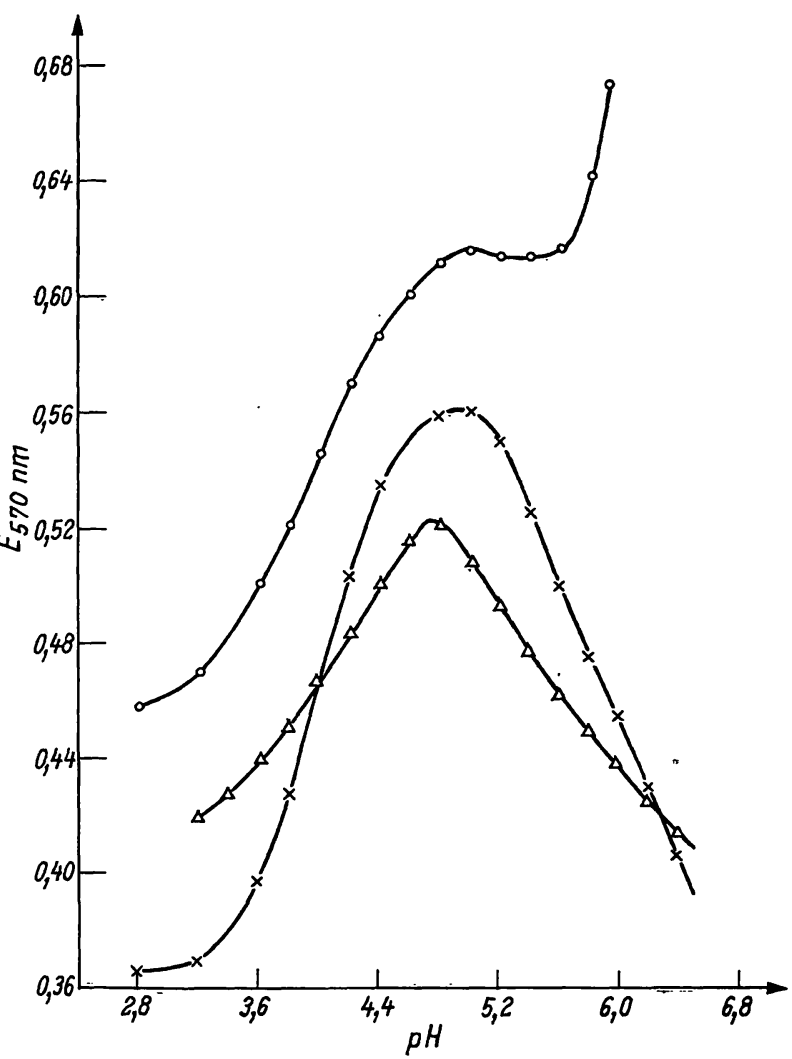

Abb. 4

pH-Aktivitätskurven der sauren Carboxypeptidase in verschiedenen Geweben $(O=$ Pankreas, $\Delta=$ Leber, $x=$ Herz) in $0,1 M$ CitratPuffer. Die Enzymkonzentrationen wurden jeweils nur für die Werte

zeigten die Organe: Gehirn, Herz und subkutanes Bindegewebe. In Abbildung 4 ist die Aktivität der sauren Carboxypeptidase in Pankreas, Leber und Herz in Abhängigkeit vom $\mathrm{pH}$-Wert aufgetragen. Dabei zeigt sich ein $\mathrm{pH}$-Optimum zwischen $\mathrm{pH} 4,7$ und 5,0. Im Pankreasgewebe steigt die Aktivitätskurve oberhalb pH 5,2 weiter an. Papierchromatographisch findet man oberhalb $\mathrm{pH}$-Werten von 5,0 bis 5,2 freies Serin. Dies spricht für die Aktivität der Leucinaminopeptidase. Die Hydrolyse des Tripeptids Ser-Leu-Leu bei pH 4,8-5,0 kann im wesentlichen auf die Aktivität der sauren Carboxypeptidase zurückgeführt werden. Dies wird auch durch 


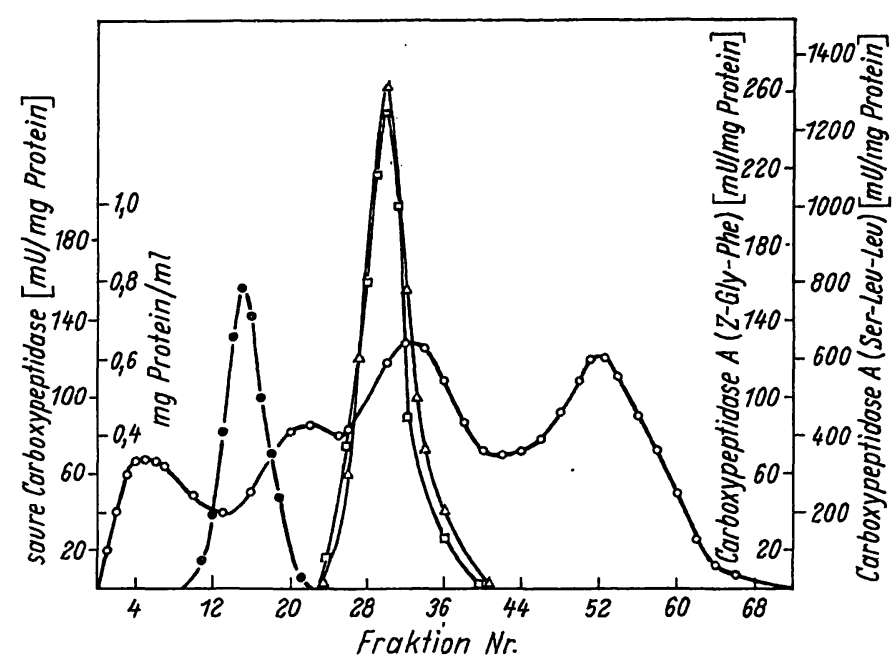

Abb. 5

Enzymaktivitäten (mU/mg Protein) von saurer Carboxypeptidase (• Substrat: Ser-Leu-Leu, pH 4,5) und von Carboxypeptidase A

Substrate: Z-Gly-Phe $\square$ und Ser-Leu-Leu $\Delta$, pH: 7,4 nach Gelfiltration der Ammoniumsulfatfraktion $11-30$ Gew. \% an Sephadex-G-150 Proteingehalt (o) in $\mathrm{mg} / \mathrm{ml}$ (Extinktionsmessung bei $280 \mathrm{~nm}$ )

eine chromatographische Reinigung an Sephadex-G-150 veranschaulicht (Abb. 5). Dabei kommt es gegenüber der Aktivität im Homogenat zu einer 40 fachen Anreicherung (Tab. 4). Im Bereich der bei pH 4,5 Ser-LeuLeu spaltenden Carboxypeptidase werden bei gleichem $\mathrm{pH}-$ Wert in keinem meßbaren Umsatz die Substrate des Kathepsins A, der Carboxypeptidase A und der katheptischen Carboxypeptidasen: Z-Glu-Tyr und Z-Gly-Phe a ngegriffen.

Tab. 4

Reinigung der sauren Carboxypeptidase aus menschlichem Pankreasgewebe

\begin{tabular}{lcc}
\hline \multicolumn{1}{c}{ Reinigung } & spezif. Aktivität (mU/mg) & Anreicherung \\
\hline $\begin{array}{l}\text { Homogenat } \\
\left(\mathrm{NH}_{4}\right)_{2} \mathrm{SO}_{4}-\text {-Fällung }\end{array}$ & 3,9 & 1 \\
$11-30$ Gew. & 16,0 & 4 \\
$\begin{array}{l}\text { Gel-Filtration } \\
\text { an Sephadex-G-150 }\end{array}$ & 158,0 & 40 \\
(Aktivitätsgipfel) & & \\
\hline
\end{tabular}

$\mathrm{Da}$ bei $\mathrm{pH}$ 7,4 im Bereich der sauren Carboxypeptidase kein Ser-Leu-Leu in meßbarer Größenordnung gespalten wird, kommt auch die Pankreascarboxypeptidase B für die Spaltung dieses Tripeptids nicht in Betracht. Mittels Papierchromatographie konnte die carboxylendständige Leucinabspaltung von Ser-Leu-Leu bei pH 4,5 - damit die Esxitenz der sauren Carboxypeptidase in menschlichen Geweben nachgewiesen werden. Auf Grund der Spaltung von Z-Gly-Phe bei pH 7,4 konnte die Pankreascarboxypeptidase A identifiziert und deutlich von der sauren Carboxypeptidase abgetrennt werden. Sie spaltet bei gleichem $\mathrm{pH}$-Wert das Substrat Ser-Leu-Leu mit wesentlich höherer Geschwindigkeit als das Substrat Z-Gly-Phe. Der Nachweis erfolgt quantitativ nach der bei l. c. (18) angegebenen Methodik und qualitativ papierchromatographisch durch Identifizierung des abgespaltenen carboxylendständigen Leucin bzw. Phenylalanin. Gleichzeitig wird die Interferenz der Leucinaminopeptidase ausgeschlossen, da in diesem Bereich bei $\mathrm{pH} 7,4$ kein aminoendständiges

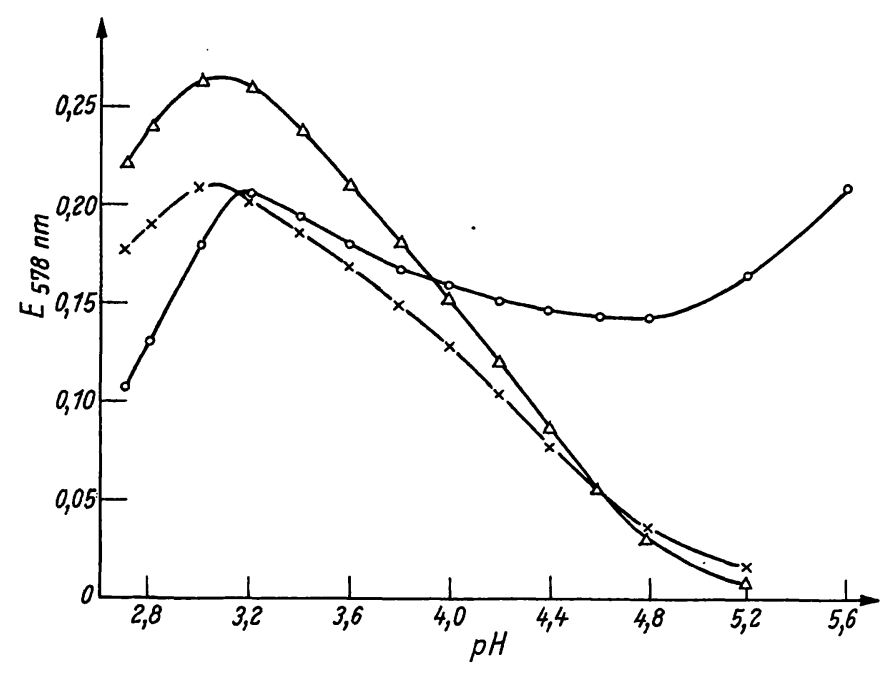

Abb. 6

pH-Aktivitätskurve des Kathepsins aus verschiedenen Geweben $(\circ=$ Pankreas, $\Delta=$ Leber, $\quad x=$ Herz). Inkubationsansätze mit jeweils nur für die Werte eines Organs mit gleicher Aktivität eingesetzt

Serin des Testsubstrates Ser-Leu-Leu in nachweisbarer Größenordnung abgespalten wird. Die pH-Optima des Kathepsins liegen in den untersuchten Geweben zwischen 3,0 und 3,5 (Abb. 6).

\section{Diskussion}

Zur Aufrechterhaltung einer konstanten Mucopolysaccharid-Protein-Konzentration in menschlichen Geweben müssen Synthese- und Abbauvorgänge streng korreliert ablaufen. Mittels radioaktiv markierter Substanzen $\left({ }^{35} \mathrm{SO}_{4},{ }^{14} \mathrm{C}\right.$-Acetat, ${ }^{14} \mathrm{C}$-Glucose, ${ }^{14} \mathrm{C}$-Serin u. a.) konnten zahlreiche Schritte in der Synthese verfolgt werden (10, 11,23). Von großem Interesse war sowohl das Verhalten beider Komponenten im Stoffwechsel als auch die Frage nach der an der Bindung zwischen Mucopolysacchariden und Proteinkomponenten beteiligten Substrate. Untersuchungen von Gross, Matheivs und DorfimaN (24) am Rippenknorpel von Ratten geben Hinweise dafür, daß der Mucopolysaccharid-Proteinkomplex als Einheit den Stoffwechselbedingungen unterliegt. Die Arbeiten von Hilz, KLEINE und KIRSIG (23) an KälberRippenknorpel sprechen dafür, daß die Kettenverlängerung der Polysaccharidkomponente mit ihrer Sulfatierung gekoppelt ist. Greiring und Stuhlsatz (10) zeigten in ,in vitro"-Versuchen an Rinder-Cornea, daß der Einbau von ${ }^{35} \mathrm{SO}_{4}$ in Chondroitin-4-Sulfat vom Molekulargewicht und vom Sulfatierungsgrad abhängig zu sein scheint. Salmon und Daughaday (25) konnten nachweisen, daß Aminosäuren die Aufnahme von Sulfat in Rippenknorpel stimulieren, - möglicherweise ein Hinweiș dafür, daß für die Chondroitin-SulfatSynthese gleichzeitig die Synthese der Proteinkomponente nötig ist. Auf Grund der chemischen Analysen sehen GreILING und Mitarbeiter die Verknüpfung des Chondroitin-4-Sulfat-Peptids der Cornea in einer Bindung des Serins und des Threonins mit dem GalaktosylXylosyl-Rest. Während für die Binding von Chondroitin-4-Sulfat an Protein im Rindernasenknorpel von 


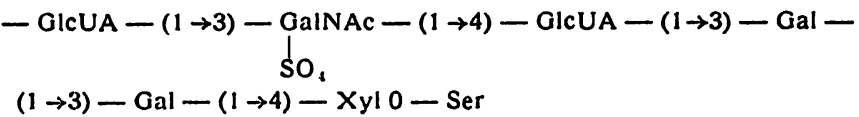

Abb. 7

Binciung von Chondroitin-4. sulfat an Protein

RODÉN und SMITH (26) die in Abbildung 7 dargestellte Struktur vorgeschlagen wurde, sehen LiNDAHL und Mitarbeiter (27) für den Heparin-Proteinkomplex aus der Darmschleimhaut des Schweins die in Abbildung 8

$$
\begin{aligned}
& \mathrm{GlCUA}-\mathrm{GICN}-\mathrm{GlCUA}-\mathrm{Gal}-\mathrm{Gal}-\mathrm{Xyl}-\mathrm{Ser}-[\text { PROTEIN } \\
& \text { Abb. } 8 \\
& \text { Bindung von Heparin an Protein }
\end{aligned}
$$

aufgezeichnete Struktur. Die Art der Bindung zwischen Hyaluronat und Protein ist noch weitgehend unbekannt.

Untersuchungen über den Wirkungssynergismus Mucopolysaccharid-Protein-abbauender Enzyme wurden von der Arbeitsgruppe um Buddecke (28) am Arteriengewebe des Rindes durchgeführt. In den vorliegenden Untersuchungen wurde erstmals die Existenz der am Abbau der Mucopolysaccharid-Protein-Komplexe synergistisch wirkenden Enzyme in zahlreichen menschlichen Geweben nachgewiesen (siehe auch bei PLATT und Hartmann (21) und Stein und Platt (22). Die Aktivitätsmessungen der Glycosaminoglycano-Hydrolasen und der Peptidhydrolasen wurden an natürlichen und synthetischen Substraten durchgeführt. Durch die synergistische Wirkung dieser 5 lysosomalen Enzyme kommt es zu einer Depolymerisietung des HyaluronatProtein-Komplexes (Abb. 1).

Uber die biologische Bedeutung der hohen Glycosaminoglycano-Hydrolasenaktivitäten in den Organen des retikulo-hystiocytären Systems (Leber, Milz und Thymus) läßt sich zur Zeit ebenso wenig sagen wie über die hohen sauren Carboxypeptidase- und Kathepsin-Aktivitäten in der Nebenniere.

Um die Entstehung von Peptiden - durch endogene Proteinspaltung - als mögliche Substrate der sauren Carboxypeptidase gering zu halten, wurde vor den Enzymaktivitätsmessungen eine fraktionierte Ammoniumsulfatfällung durchgeführt. Auf Grund von Abbauversuchen der Insulin-B-Kette und des Chondroitin-SulfatProteins konnte in vitro die synergistische Wirkung zwischen Kathepsin D und der sauren Carboxypeptidase gezeigt werden $(24,19)$. Die vorliegenden Messungen zeigen - bis auf das Thymusgewebe - ein gleichsinniges Aktivitätsverhalten der sauren Carboxypeptidase und des Kathepsin D in den von uns untersuchten Organen. Der Beweis für eine synergistische Wirkung ,in vivo" konnte bisher nicht erbracht werden. Ein Hinweis für einen Synergismus in der Zelle könnte jedoch sein, daß beide Enzyme lysosomaler Herkunft sind und beide im sauren $\mathrm{pH}-$ Bereich spalten, wobei das $\mathrm{pH}-\mathrm{Op}$ timum des Kathepsins je nach Substrat variiert (29).

\section{Literatur}

1. Meyer, K., in: W. H. Hauss und H. Losse, II. Sympos. an der Med. Univ. Klin. Münster, Georg Thieme, Stuttgart (1960). 2. Matheivs, M. B., Arch. Biochem. Biophysics 74, 158 (1958). 3. Buddecke, E., und M. Schubert, Hoppe-Seyler's Z. physiol. Chem. 325, 189 (1961). - 4. Johnson, B. und M. SChubert, J. Clin. Invest. 39, 1752 (1960). - 5. Shatron, J. und M. Schubert, J. biol. Chemistry 211, 565 (1954). - 6. Yu, S. und H. BlumenTHAL, J. Gerontol. 13, 366 (1958). - 7. Frimmer, W. und E. BUDdeCKe, Z. Naturforsch. 196, 790 (1964). - 8. BHATNAGER, R. S. und D. J. Prockop, Biochim. biophysics Acta Amsterdam 130, 383 (1966). - 9. Dohlmann, C. H. und H. Boström, Acta Ophthalmol. (Copenhagen) 33, 455 (1955). - 10. GrerLING, H., H. W. Stuhlsatz und L. Plagemann, Hoppe-Seyler's Z. physiol. Chem. 34S, 121 (1967). - 11. KLeIne, T. O. und H. Hilz, HoppeScyler's Z. physiol. Chem. 349, 1027 (1968). - 12. Wortaran, B. und J. L. Strominger, Amer. J. Ophthalmol. 44, 291 (1957). 13. Weichiselbaum, T. E., Amer. J. Clin. Path. 16, 40 (1946). 14. Loivry, O. H., N. J. Rosebrough, A. L. Farr und R. J. Randall, J. biol. Chemistry 193, 265 (1951). - 15. Morgan, W. T. J. und L. A. Elson, Biochem. J. 28, 988 (1934). - 16.
Bernfeld, P. und W. H. Frshman, J. biol. Chemistry 202, 757 (1953). - 17. Pugh, D., D. H. LeABACK und P. G. Walker, Biochem. J. 65, 464 (1957). - 18. BuDDECKE, E., G. REICH und U. Stein, Hoppe-Seyler's Z. physiol. Chem. 347, 192 (1966). 19. Stein, U., U. Weaer und E. BudDECKE, Hoppe-Seyler's Z. physiol. Chem. 349, 349 (1968). - 20. Anson, M. L., J. Gen. Physiol. 22, 79 (1938). - 21. Platt, D. und R. Hartmann, Klin. Wschr. 45, 998 (1967). - 22. Stein, U. und D. Platt, Klin. Wschr. 46, 1145 (1968). - 23. KLeINE, T. O., H. J. Kirsig und H. Hrzz, Hoppe-Seyler's Z. physiol. Chem. 349, 1037 (1968). 24. Gross, J.I., M. B. Mathews und A. Dorfaran, J. biol. Chemistry 235, 2889 (1960). - 25. SALMON, W: D. jr. und W. H. Daughaday, J. Laborat. Clin. Med. S. Louis 51, 167 (1958). 26. RoDÉN, L. und R. SMITH, J. biol. Chemistry 241, 5949 (1966). 27. LINDAHL, U. und L. RoDÉN, J. biol. Chemistry 241, 65 (1966). 28. Held, E., O. Hoefele, U. Stein, E. Werries und E. BudDECKE, diese Z. 6, 244 (1968). - 29. KREGAR, J., V. TURK und D. Lebez, Enzymologia 33, 80 (1967). - 30. LindAHL, U., Biochim. biophysica Acta, Amsterdam 130, 368 (1966).

\section{Dr. med. Dieter Platt Medizinische Univ.Klinik 63 Gießen \\ Klinikstr. 32b \\ Dr. med. Ulf Stein Medizinische Univ.Klinik 78 Freiburg/Brsg.}

